\title{
Directional x-ray dark-field imaging of strongly ordered systems
}

\author{
Torben Haugaard Jensen, ${ }^{1}$ Martin Bech, ${ }^{2}$ Irene Zanette, ${ }^{3}$ Timm Weitkamp, ${ }^{3}$ Christian David, ${ }^{4}$ Hans Deyhle,,${ }^{5,6}$ \\ Simon Rutishauser, ${ }^{4}$ Elena Reznikova, ${ }^{7}$ Jürgen Mohr ${ }^{7}$ Robert Feidenhans' $1,{ }^{1}$ and Franz Pfeiffer ${ }^{2}$ \\ ${ }^{1}$ Niels Bohr Institute, University of Copenhagen, 2100 Copenhagen, Denmark \\ ${ }^{2}$ Department of Physics, Technical University of Munich, 85747 Garching, Germany \\ ${ }^{3}$ European Synchrotron Radiation Facility, 38043 Grenoble, France \\ ${ }^{4}$ Paul Scherrer Institut, 5232 Villigen PSI, Switzerland \\ ${ }^{5}$ Biomaterials Science Center, University of Basel, 4031 Basel, Switzerland \\ ${ }^{6}$ Institute for Materials Science, School of Dental Medicine, University of Basel, 4031 Basel, Switzerland \\ ${ }^{7}$ Karlsruhe Institute of Technology, Institute for Microstructure Technology, 76021 Karlsruhe, Germany
}

(Received 11 August 2010; published 6 December 2010)

\begin{abstract}
Recently a novel grating based x-ray imaging approach called directional x-ray dark-field imaging was introduced. Directional x-ray dark-field imaging yields information about the local texture of structures smaller than the pixel size of the imaging system. In this work we extend the theoretical description and data processing schemes for directional dark-field imaging to strongly scattering systems, which could not be described previously. We develop a simple scattering model to account for these recent observations and subsequently demonstrate the model using experimental data. The experimental data includes directional dark-field images of polypropylene fibers and a human tooth slice.
\end{abstract}

DOI: 10.1103/PhysRevB.82.214103

PACS number(s): 87.59.-e, 07.85.-m, 62.20.-x, 78.70.Ck

\section{INTRODUCTION}

Grating-based $\mathrm{x}$-ray imaging has recently been used to demonstrate a variety of different contrast mechanisms. ${ }^{1-17}$ These include phase contrast imaging, ${ }^{1-8}$ dark-field imaging, $, 4,7,-16$ and lately also directional dark-field imaging (DDFI). ${ }^{17}$ The latter provides information on structures in the sample on length scales smaller than the actual image resolution. The method can be used to map, e.g., local fiber orientations and has been demonstrated in a setup using a conventional $\mathrm{x}$-ray tube. DDFI analyzes the angular variation in the scattering signal. In the first presentation of the method by Jensen $e t a l .{ }^{17}$ the visibility variations were described by a Fourier series expansion up to the first order, i.e., by a sinusoidal behavior.

$$
V(j, k, \omega) \approx b_{0}(j, k)+b_{1}(j, k) \cos \left(2\left[\omega-\psi_{1}(j, k)\right]+\pi\right) .
$$

In this paper we will extend our previously introduced theoretical basis and data interpretation schemes for DDFI to strongly ordered systems. This becomes necessary as strongly ordered systems produce highly eccentric scattering patterns, which can no longer be sufficiently described by the previously introduced sinusoidal data interpretation for DDFI.

\section{EXPERIMENTAL SETUP}

The experimental setup is shown in Fig. 1. The grating interferometer has previously been described in detail. ${ }^{3,5,6,9,10,17}$ It consists of a phase grating (G1) and an analyzer absorption grating (G2). The first grating, (G1) generates a periodic intensity modulation at the position of the second grating (G2). The second grating is used to analyze the position and amplitude of the intensity modulation. When the phase grating is scanned in the direction perpendicular to the optical axis and the grating lines, the intensity signal $I(j, k)$ for each pixel with coordinates $(j, k)$ will oscillate as a function of grating position $x_{g}$ [Fig. 1(b)]. Previous work has demonstrated how the intensity can be well described by a first-order Fourier expansion ${ }^{4,9}$

(a)



(b)

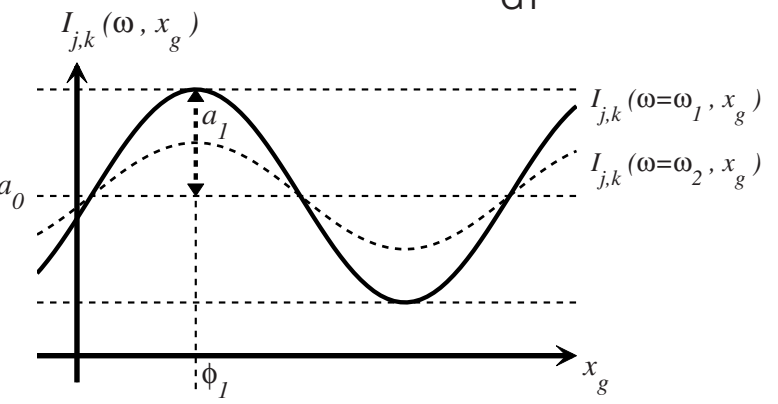

FIG. 1. (Color online) X-ray grating interferometer. (a) Setup with a phase grating G1 and an analyzer absorption grating G2. The sample is rotated around the optical axis. (b) For each angular position, the grating G1 is scanned along $x_{g}$, yielding an intensity modulation $I_{j, k}\left(\omega, x_{g}\right)$ for each pixel $(j, k)$ on the two-dimensional image detector. 


$$
I\left(j, k, \omega, x_{g}\right) \approx a_{0}(j, k, \omega)+a_{1}(j, k, \omega) \cos \left[\frac{2 \pi}{g_{2}} x_{g}-\phi_{1}(j, k, \omega)\right],
$$

where $g_{2}$ is the period of $\mathrm{G} 2$ and $\omega$ the rotation angle of the sample around the optical axis.

Scattering in the sample will reduce the oscillation amplitude. The dark-field scatter signal is called the visibility and describes the reduction in oscillation amplitude. The visibility is defined as

$$
V(j, k, \omega) \equiv \frac{a_{1}^{s}(j, k, \omega) / a_{0}^{s}(j, k, \omega)}{a_{1}^{r}(j, k) / a_{0}^{r}(j, k)} .
$$

The superscripts $s$ and $r$ denote the values measured with the specimen in place $\left({ }^{s}\right)$ and as a reference without $\left({ }^{r}\right)$. The visibility, $V(j, k, \omega)$ is an inverse measure of the effective integrated local small- (and ultrasmall-) angle scattering power of the sample. ${ }^{7,9,10,17}$ It will have a value close to 1 for samples with negligible scattering. For strongly scattering samples the visibility will be reduced yielding values of $V$ $<1$.

As described by Jensen et al. ${ }^{17}$ the dark-field contrast arises due to the component of the scattering that happens perpendicular to the grating lines. The scattering component parallel to the grating lines will produce only negligible dark-field contrast. In the following we will develop a model to describe how the dark-field signal varies as a function of angle.

\section{MODEL}

We will begin by assuming that the scattering from a single point on the sample will result in a 2D Gaussian scattering intensity profile, $S_{2}$ at the plane of G2. This assumption of a Gaussian scattering distribution corresponds well to assumptions made when using the dark-field signal for tomography. ${ }^{13-15}$ Let the scattering profile $S_{2}$ be defined as follows:

$$
\begin{gathered}
S_{2}(x, y)=\frac{1}{2 \pi \sigma_{1} \sigma_{2}} \exp \left[-\left(a x^{2}+2 b x y+c y^{2}\right)\right], \\
a:=\frac{\cos \left(\psi_{1}\right)^{2}}{2 \sigma_{1}^{2}}+\frac{\sin \left(\psi_{1}\right)^{2}}{2 \sigma_{2}^{2}}, \\
b:=\sin \left(\psi_{1}\right) \cos \left(\psi_{1}\right)\left(-\frac{1}{2 \sigma_{1}^{2}}+\frac{1}{2 \sigma_{2}^{2}}\right), \\
c:=\frac{\sin \left(\psi_{1}\right)^{2}}{2 \sigma_{1}^{2}}+\frac{\cos \left(\psi_{1}\right)^{2}}{2 \sigma_{2}^{2}} .
\end{gathered}
$$

$S_{2}$ is a 2D Gaussian distribution with the width $\sigma_{1}$ and $\sigma_{2}$ in the two axial directions, and the first axis rotated by the angle $\psi_{1}$. An example of how $S_{2}(x, y)$ could look like for a given $\sigma_{1}, \sigma_{2}$, and $\psi_{1}$ is shown in Fig. 2. Since the gratings are only sensitive to scattering perpendicular to the grating lines, the dark-field signal will be measuring the projection

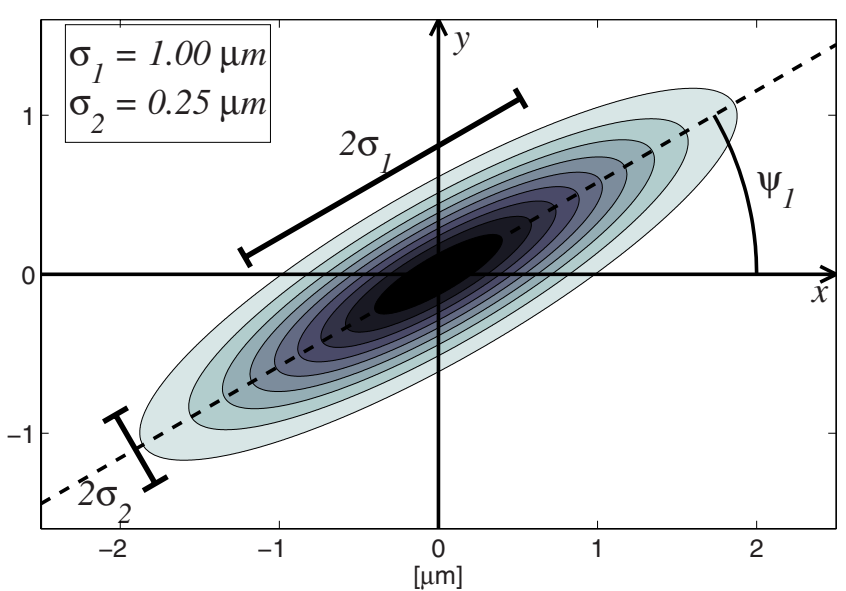

FIG. 2. (Color online) Illustration of 2D Gaussian scattering function, $S_{2}(x, y)$, with $\psi_{1}=30^{\circ}$.

of $S_{2}$ onto the $x$ axis. This corresponds to integrating $S_{2}$ along $y$

$$
\begin{gathered}
S(x)=\frac{1}{\sqrt{2 \pi \sigma^{2}}} \exp \left(\frac{-x^{2}}{2 \sigma^{2}}\right), \\
\sigma^{2}=\frac{1}{2}\left(\sigma_{1}^{2}+\sigma_{2}^{2}\right)+\frac{1}{2}\left(\sigma_{1}^{2}-\sigma_{2}^{2}\right) \cos \left(2 \psi_{1}-\pi\right) .
\end{gathered}
$$

The projection of $S_{2}$ is thus another Gaussian, $S$, with the width $\sigma$. As mentioned above, the variations in intensity in a pixel can be described as follows: $I^{r}(x)=a_{0}+a_{1} \cos \left(\frac{2 \pi}{g_{2}} x\right.$ $\left.-\phi_{1}\right)$. Since the scattering from a single point is described by $S(x)$ at the position of the second grating, the perturbed intensity variation can be found as the convolution of the original intensity function, $I^{r}(x)$ and the scattering function, $S(x)$.

$$
\begin{gathered}
I^{S}(x)=I^{r}(x) \otimes S(x), \\
=a_{0}+a_{1} \exp \left(\frac{-2 \pi^{2} \sigma^{2}}{g_{2}^{2}}\right) \cos \left(\frac{2 \pi}{g_{2}} x-\phi_{1}\right) .
\end{gathered}
$$

Recalling the definition of the visibility from Eq. (3) and combining with Eq. (6) we see that the visibility varies as

$$
\begin{aligned}
V\left(\psi_{1}\right)= & \exp \left[\frac{-\pi^{2}}{g_{2}^{2}}\left(\sigma_{1}^{2}+\sigma_{2}^{2}\right)\right] \exp \left[\frac { - \pi ^ { 2 } } { g _ { 2 } ^ { 2 } } ( \sigma _ { 1 } ^ { 2 } - \sigma _ { 2 } ^ { 2 } ) \operatorname { c o s } \left(2 \psi_{1}\right.\right. \\
& -\pi)]
\end{aligned}
$$

If we include the rotation of the sample, $\omega$, during measurement we can rewrite Eq. (7)

$$
\begin{aligned}
V(\omega)= & \exp \left[\frac{-\pi^{2}}{g_{2}^{2}}\left(\sigma_{1}^{2}+\sigma_{2}^{2}\right)\right] \exp \left\{\frac{-\pi^{2}}{g_{2}^{2}}\left(\sigma_{1}^{2}-\sigma_{2}^{2}\right) \cos [2(\omega\right. \\
& \left.\left.\left.-\psi_{1}\right)-\pi\right]\right\} .
\end{aligned}
$$

Equation (8) is a full description of how the visibility changes as a function of $\left(\sigma_{1}, \sigma_{2}, \psi_{1}, \omega\right)$. The detailed relationship between the local structure of the sample and the 
parameters, $\left(\sigma_{1}, \sigma_{2}, \psi_{1}, \omega\right)$ is subject to further investigations. We will now take a look at two special cases.

\section{A. Weakly oriented scatterers}

In the previous study ${ }^{17}$ it was assumed that the visibility variations were sinusoidal. From the model developed above [Eq. (8)] we know this is not generally the case. Let $K_{1}$ $\equiv \frac{\pi^{2}\left|\sigma_{1}^{2}-\sigma_{2}^{2}\right|}{g_{2}^{2}}$. The parameter $K_{1}$ describes how eccentric the scattering profile is relative to the grating period. We note that in the special case where $K_{1} \sim 0$, we can Taylor expand the second exponential of Eq. (8)

$$
\begin{aligned}
V(\omega) \approx & \exp \left[\frac{-\pi^{2}}{g_{2}^{2}}\left(\sigma_{1}^{2}+\sigma_{2}^{2}\right)\right]\left\{1-\frac{\pi^{2}}{g_{2}^{2}}\left(\sigma_{1}^{2}-\sigma_{2}^{2}\right) \cos \left[2\left(\omega-\psi_{1}\right)\right.\right. \\
& -\pi]\} .
\end{aligned}
$$

We note that in Eq. (9) the visibility varies sinusoidally. From this we can conclude that if $K_{1} \sim 0$ that is if the scattering profile has widths that are small compared to the period of the second grating, $g_{2}$, or if the difference between the widths of the scattering profile are small compared to the period of the second grating, $g_{2}$, then the visibility varies sinusoidal. So weakly scattering samples and samples that are not strongly oriented will produce sinusoidal visibility variations. The model presented here is thus consistent with the previously published methodology for samples with $K_{1}$ $\sim 0$. For samples with highly eccentric scattering profiles, the parameter $K_{1}$ will be much greater than 0 , and the visibility variations will no longer be sinusoidal.

\section{B. Unscattered photons}

In the model described above [Eqs. (8) and (9)] we have assumed that the entire beam is scattered and no part of it pass the sample without being scattered. Only the part of the beam that is scattered can contribute to the reduction in visibility so if a part of the beam passes through the sample without being scattered then the visibility cannot be reduced to zero no matter the width of the scattering profile of the scattered part of the beam. If, e.g., $40 \%$ of the beam is not scattered then the visibility will take values greater than 0.4 . Such transmission can be included in the model using a constant taking the not scattered part of the beam into account. Let $\alpha$ be the proportion of the beam that has been scattered. Then Eq. (6) is replaced by

$$
\begin{gathered}
I^{s}(x)=I^{r}(x) \otimes[(1-\alpha) \delta(x)+\alpha S(x)], \\
=a_{0}+\left[(1-\alpha)+\alpha \exp \left(\frac{-2 \pi^{2} \sigma^{2}}{g_{2}^{2}}\right)\right] \cdot a_{1} \cos \left(\frac{2 \pi}{g_{2}} x-\phi_{1}\right)
\end{gathered}
$$

and the visibility takes the form

$$
V(\omega)=(1-\alpha)+\alpha \exp \left[\frac{-2 \pi^{2} \sigma^{2}}{g_{2}^{2}}\right],
$$

$$
\sigma^{2}=\frac{1}{2}\left(\sigma_{1}^{2}+\sigma_{2}^{2}\right)+\frac{1}{2}\left(\sigma_{1}^{2}-\sigma_{2}^{2}\right) \cos \left(2\left[\omega-\psi_{1}\right]-\pi\right) .
$$

The sinusoidal behavior for $K_{1} \sim 0$ is still valid when we use Eqs. (11) and (12) to describe the visibility. With the visibility varying sinusoidally the variations can be described by the mean and oscillation amplitude, and it is thus not possible to determine three independent parameters hence $\left(\sigma_{1}, \sigma_{2}, \alpha\right)$ can no longer be uniquely determined. This does introduce an ambiguity in our model, which does not limit the ability to describe and predict visibility variations but can limit the ability to quantify the measured scattering profiles.

In the following we will present experimental data with $K_{1}>1$, where the visibility does not vary sinusoidally. The two examples described will be cases with $\alpha=1$ and $\alpha<1$, respectively. The experimental data will be well described by the model presented above.

\section{RESULTS}

\section{A. Experimental details}

The directional dark-field experiments were conducted at beamline ID19 of the European Synchrotron Radiation Facility (ESRF, Grenoble) using monochromatic x-rays at an energy of $17.6 \mathrm{keV}{ }^{18}$ The grating interferometer was placed $150 \mathrm{~m}$ from the wiggler source. The silicon phase grating was fabricated by a process involving photolithography and anisotropic wet etching into silicon. ${ }^{19}$ The absorption gold grating was fabricated by a process involving deep x-ray lithography and gold electroplating. ${ }^{20}$ The gratings periods were $\mathrm{g}_{1}=4.79 \mu \mathrm{m}$ and $\mathrm{g}_{2}=2.40 \mu \mathrm{m}$. The heights of the grating structures were $23 \mu \mathrm{m}(\mathrm{G} 1)$ and $50 \mu \mathrm{m}(\mathrm{G} 2)$. The height of G1 was optimized for $\pi$ phase shift at an X-ray energy of $17.6 \mathrm{keV}$. The distance between G1 and G2 was $447 \mathrm{~mm}$, corresponding to the eleventh fractional Talbot distance. ${ }^{8}$ The gratings were mounted with the grating lines horizontally to use the greater coherence in the vertical direction. The images were recorded using a FReLoN CCD with $2048 \times 2048$ pixels with an effective pixel size of $7.46 \times 7.46 \mu \mathrm{m}^{2}$.

Each dark-field image was generated using raw images recorded for 16 different grating position in $x_{g}$ over two periods. The dark-field signal was extracted from the intensity modulation $I\left(j, k, \omega, x_{g}\right)$ for each rotation angle using onedimensional fast Fourier transforms.

\section{B. Fibers}

We use a mesh of polypropylene fibers (PP) to illustrate the model in a case where $\alpha=1$. The experiments were made with 90 angular rotation steps over $180^{\circ}$. The exposure time for each image was $1.5 \mathrm{~s}$, resulting in a total exposure time of 36 min. In Figs. 3(a) and 3(b) we see the average visibility of the fiber mesh. In Fig. 3 we identified sets of ten distinct points with no fibers, single fibers, and coaxially overlapping pairs of fibers, respectively. In Fig. 3(b), a magnified section of Fig. 3(a), examples of spots with $0,1,2$, and several fibers are marked. In the images we clearly see that point (b) is placed in the middle of one fiber and point (c) is placed 

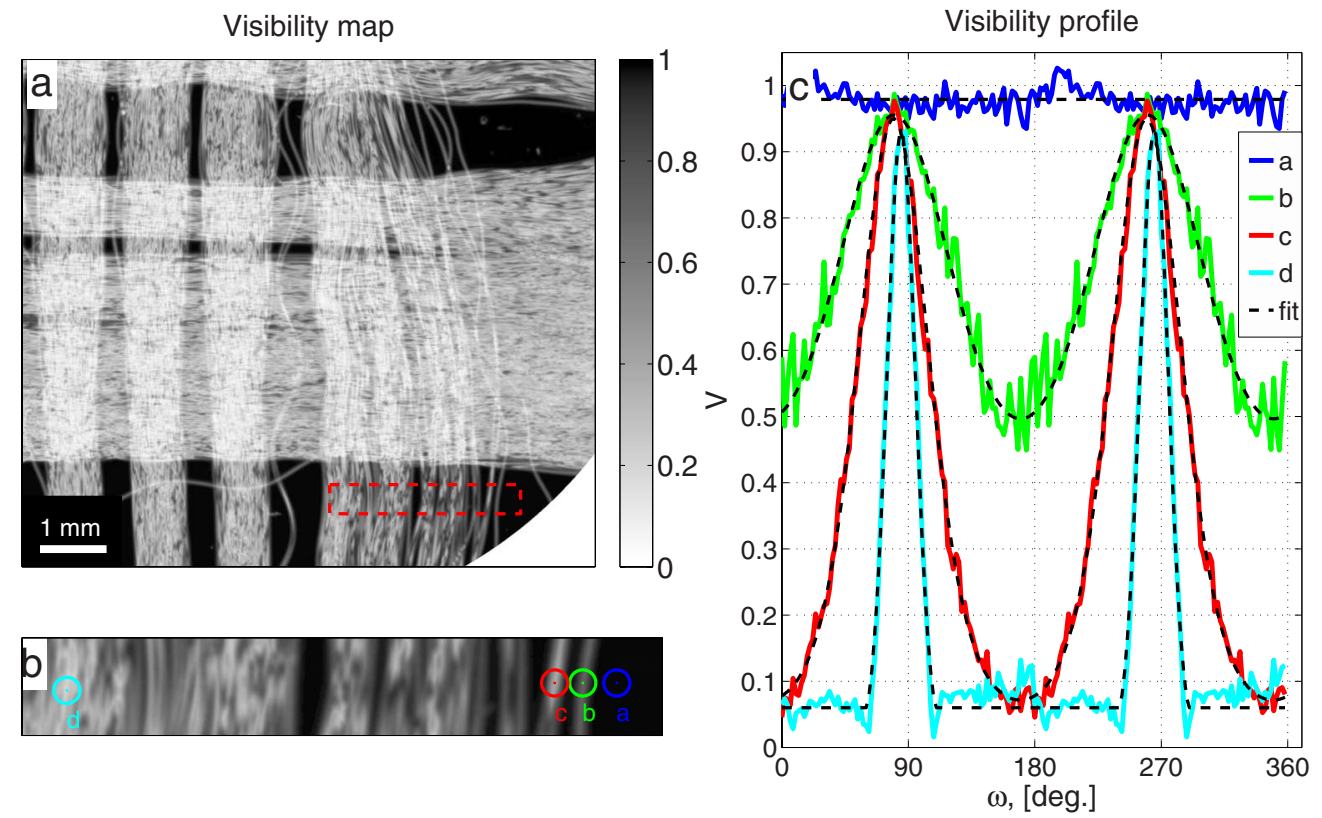

FIG. 3. (Color online) (a) Average visibility of a polypropylene fiber mesh. (b) Enlargement of part of (a), height 0.5 mm. (c) Visibility variation as a function of rotation angle for four different points marked in (b). Note the nonsinusoidal behavior of the read and cyan lines. The dashed black lines are model fits to the measured values.

where two fibers overlap. The fiber diameters are $32 \mu \mathrm{m}$ and the pixel size is $7.46 \mu \mathrm{m}$. It is thus possible to effectively probe only $\mathrm{x}$-rays that have passed through the middle of one or two fibers.

The visibility variation in the four points marked in Fig. 3(b) are plotted in Fig. 3(c). Upon inspection we see great variation in the visibility curves for the four different spots. We also note that some of these visibility variations are clearly not sinusoidal. The visibility variations for the four shown points as well as the 30 other identified points have been thoroughly analyzed using the model we developed above [Eq. (8)]. For all the points the visibility variations were fitted using the parameters $\sigma_{1}, \sigma_{2}$, and $\psi_{1}$. We see no effects from the not scattered part of the beam (except in points with no fibers). The parameter $\alpha$ is thus set to $\alpha=1$ for all fits. In Fig. 3(c) the fits are plotted for the four curves with black dashed lines. We see that the model described above can be used to accurately fit the experimentally recorded visibility variations. ${ }^{21}$ The fitted parameters for all points are printed in Table I. Along with the fitted parameters $\sigma_{1}, \sigma_{2}$, and $\psi_{1}$, the derived parameter $K_{1}$ and the standard $\mathrm{x}$-ray absorption for all points are also printed in the table. The 30 points were analyzed to give an estimate of the uncertainty in the measurements. The noted uncertainty for the associated fitted parameters are the standard deviations over ten points. Note that the momentum transfer values that are involved in the generation of the dark-field signal are below the well-known small-angle $\mathrm{x}$-ray scattering regime. Upon analysis of the fitted parameters we see that the width of two fibers [as in point (c)] is double that of the width of one fiber [as in point (b)]. Once the magnitude of the widths of the scattering profiles has been calibrated we see that with excellent signal-to-noise ratio we can distinguish one and two fibers. Note this is not possible with the absorption information in this case, where the signal-to-noise ratio is too low to infer usable information. The noise is also the origin of the negative absorption in the point without a fiber. In point (d) multiple fibers are overlapping. We see that there is a negli-

TABLE I. Fitted parameters for points with $0,1,2$, and several overlapping fibers. The fits were made using Eq. (8). Included are also the derived parameter $K_{1}$ as well as the measured absorption.

\begin{tabular}{lcccccc}
\hline \hline Name & Fibers & \multicolumn{2}{c}{ Fitted parameters } & Meas. param. & Deriv. param. \\
\hline & & $\sigma_{1}$ & $\sigma_{2}$ & $\psi_{1}$ & Absorption & $K_{1}$ \\
& $10 \times 0$ & $0.06 \pm 0.004 \mu \mathrm{m}$ & $0.06 \pm 0.004 \mu \mathrm{m}$ & 0 & $0.6 \pm 0.4 \%$ & 0 \\
& $10 \times 1$ & $0.46 \pm 0.02 \mu \mathrm{m}$ & $0.11 \pm 0.01 \mu \mathrm{m}$ & - & $1.5 \pm 0.5 \%$ & 0.3 \\
& $10 \times 2$ & $0.87 \pm 0.04 \mu \mathrm{m}$ & $0.13 \pm 0.03 \mu \mathrm{m}$ & - & $2.3 \pm 0.8 \%$ & 1.3 \\
(a) & 0 & $0.08 \mu \mathrm{m}$ & $0.08 \mu \mathrm{m}$ & $0.0^{\circ}$ & $-0.2 \%$ & 0 \\
(b) & 1 & $0.45 \mu \mathrm{m}$ & $0.12 \mu \mathrm{m}$ & $169.9^{\circ}$ & $1.2 \%$ & 0.3 \\
(c) & 2 & $0.88 \mu \mathrm{m}$ & $0.12 \mu \mathrm{m}$ & $169.5^{\circ}$ & $2.8 \%$ & 1.3 \\
(d) & Multiple & $2.19 \mu \mathrm{m}$ & $0.14 \mu \mathrm{m}$ & $174.9^{\circ}$ & $5.5 \%$ & 8.2 \\
\hline \hline
\end{tabular}


gible increase in $\sigma_{2}$ for that point. So we can conclude that all of the fibers overlap coaxially and thus produce no scattering in the direction perpendicular to their axes.

In the presentation of the model above, we developed a criteria $\left(K_{1} \sim 0\right)$ for when our model predicted the previously described sinusoidal behavior. The parameter $K_{1}$ is tabulated in Table I. In Fig. 3 we note that for one fiber the visibility variation is sinusoidal as reported in Jensen et al. ${ }^{17}$ while for two and more fibers the visibility clearly does not vary sinusoidally. For the points with one fiber we note that $K_{1}=0.3$ $\sim 0$ which was the criteria for sinusoidal behavior. For two and multiple fibers we have that $K_{1}>1$ as expected for nonsinusoidal behavior. The observations in Fig. 3(c) thus corresponds well with our discriminatory parameter, $K_{1}$. We can conclude that not all of the experimental data presented here could satisfactorily have been described by simple sinusoidal behavior. Our model however provides an accurate description of the variations.

The results clearly demonstrate that for strongly oriented samples the sinusoidal approach is no longer suitable for describing the visibility variations. These fibers were in a simple mesh. Since we use X-rays for this investigation, the method would have worked equally well if the fibers had been embedded. The method could thus be used for quality control for fiber reinforced materials.

Besides using the model for determining the location, orientation, and number of overlapping fibers in each point, the model presented here could be used to design optimal grating parameters if interested in a specific problem. If the Talbot distance is doubled, the width of the Gaussian scattering function on the detector will double. The effect of a change in Talbot distance can thus be predicted using the model. A second important factor is the energy. A change in energy will both influence the width of the scattering function on the detector and also reduce the scattering cross section. The changes in energy and Talbot distance could be tailored to provide a suitable use of the dynamic range of the visibility for a given task.

\section{Tooth}

We use a second sample to illustrate the extended version of the model with $\alpha<1$. The sample is a vertical slice of a human tooth. ${ }^{22}$ The experiments were made with 128 angular rotation steps over $360^{\circ}$. The exposure time for each image was $1.5 \mathrm{~s}$, resulting in a total exposure time of $51 \mathrm{~min}$. The results are shown in Fig. 4. In Fig. 4(a) the x-ray transmission is shown outlining the enamel, dentin, and pulp cavity of the tooth. Dark-field images of the visibility for three different orientations are shown in Fig. 4(b), whiter represents stronger scattering. Note that the dentin has the strongest dark-field signal. In the dentin narrow dark bands displays small areas of low scattering for the different orientations (most clearly visible near the red circle for $\omega=0^{\circ}$ ). In Fig. 4(c) the visibilities for the three points marked in Fig. 4(b) are plotted. Narrow peaks of high visibility show that the scattering is strongly oriented. The peaks are located at different angles, corresponding to different orientation of the scattering structures in the dentin in each of the three points.
The fits made using the extended model [Eqs. (11) and (12)] are plotted as dashed black lines. We see that the baselines vary and are greater than 0 . The narrow peaks and the nonzero baseline show that a part of the beam has not been scattered. This partially scattered beam indicates that $\alpha<1$ and the extended model is needed to describe the observed visibility changes. The variation in baseline shows that a different proportion of the beam is unscattered through different parts of the tooth.

Some of the visibility curves show more than two peaks. The multiple peaks could be caused by the overlapping of different structures due to the thickness of the sample $(500 \mu \mathrm{m})$. In our analysis we have focused on the most pronounced peaks for each point.

We believe that the dentin tubuli are the origin of the scattering signal. The sample was dry when measured, and the empty tubuli hence will produce a very strong smallangle scattering signal. It is also possible to use the widths of the fitted Gaussian scattering profile to estimate the $q$-space scattering vector they correspond to. For the dentin the Gaussian has a fitted major (minor) axis on G2 of $\sim 11(\sim 0.5) \mu \mathrm{m}$ (data not shown). One can use this and the G1-G2 distance to calculate the corresponding reciprocal scattering vector. The reciprocal scattering vectors corresponds to a real-space distance of $\sim 2.5(\sim 50) \mu \mathrm{m}$. We recognize the shorter distance to be close to the known size of the dentin tubuli while the long distance corresponds to the coherence length of the beam, which is thus the limiting factor for the narrow part of the scattering function. This supports the notion that the tubuli are the origin of the scattering signal.

The fits shown in Fig. 4(c) were made for all points on the sample. In Fig. 4(d) the parameter $\alpha$ is plotted showing the part of the beam that has been scattered. Note how the scattering decreases away from the pulp cavity. This corresponds well with the known property that the tubuli exists in higher concentrations closer to the pulp cavity. Note also that we can detect little or no scattering in the enamel and near the dentin enamel junction. In Fig. 4(d) points with no sample have been colored black for clarity.

The local eccentricity $\left[e \equiv\left(\sqrt{\sigma_{1}^{2}-\sigma_{2}^{2}}\right) / \sigma_{1}\right]$ is plotted in Fig. 4(e). The eccentricity shows the dentin is most strongly aligned close to the pulp. The eccentricity has values up to more than 30 . This indicates a very strong preferred orientation and correlates well with the observation of the very narrow peaks in the visibility in Fig. 4(c) and the narrow dark bands in Fig. 4(b). Only when the tubuli are aligned with in a couple of degrees of parallel with the grating lines do they produce no signal in the visibility.

The eccentricity has in Fig. 4(f) been combined with the ordering direction of the tubuli in the dentin. The color wheel shows the orientation of tubuli in the dentin, corresponding to the position of the peak $\left(+90^{\circ}\right)$ in the visibility in Fig. 4(c). In the figure the eccentricity is mapped onto the intensity of the colors. The orientation we see in Fig. 4(f) corresponds to the dentin tubuli which are mainly oriented radially away from the pulp cavity. For both Figs. 4(e) and 4(f) only points with $\alpha>0.15$ have been plotted for clarity, thus omitting pixels where only a small part of the beam has been scattered. The orientation in the enamel is not observable in the presented measurements. 
a

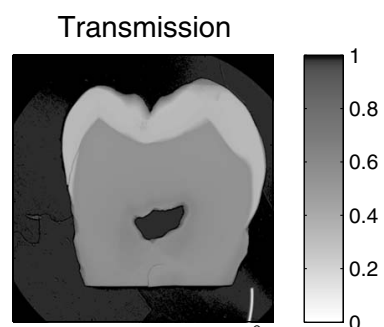

Dark field, $\omega=45^{\circ}$


d



b

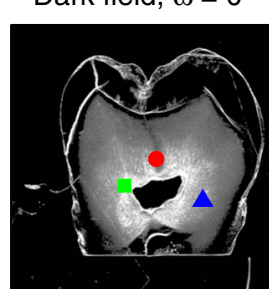

Dark field, $\omega=90^{\circ}$
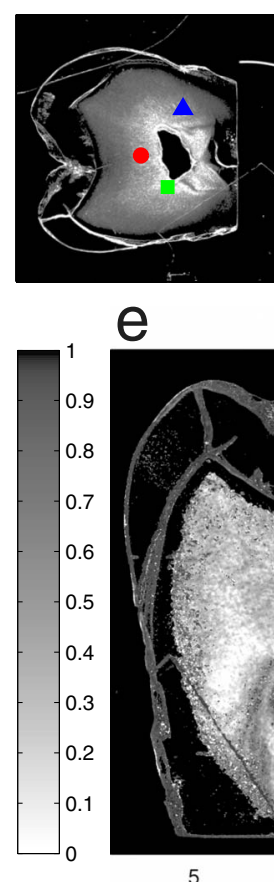

e



Visibility, $\mathrm{V}(\omega)$

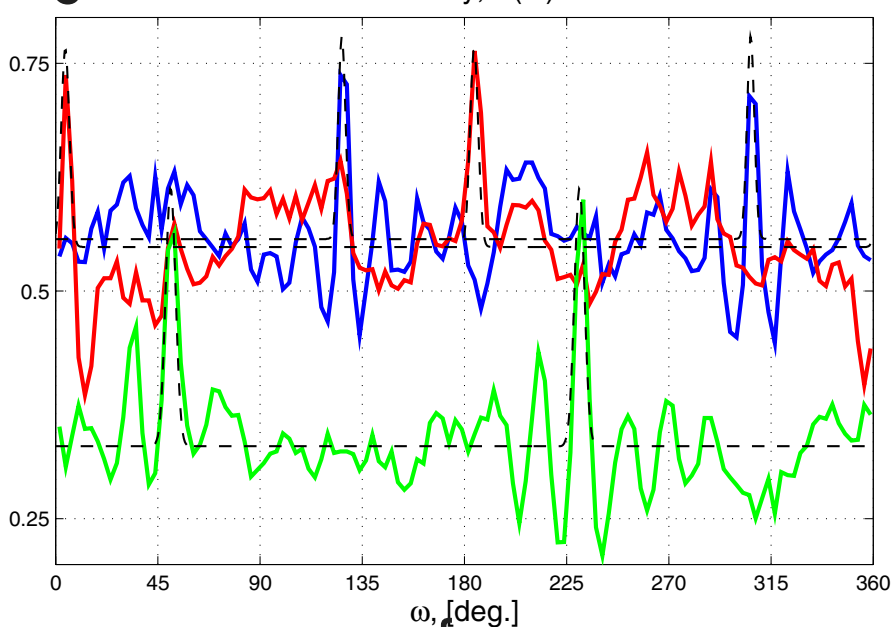

DDF

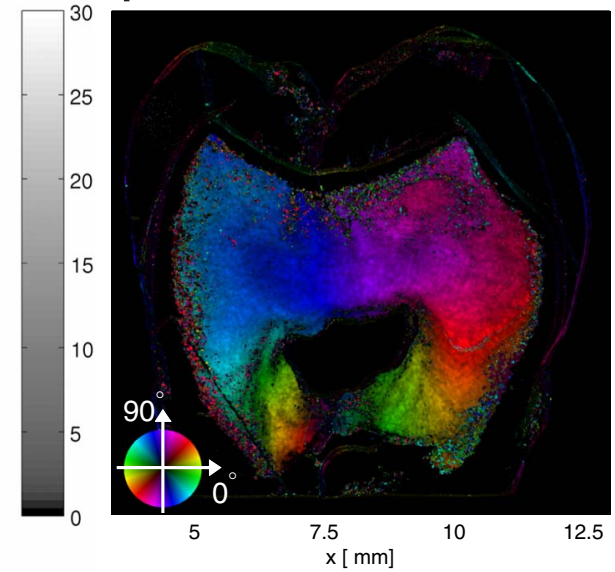

FIG. 4. (Color online) Imaging of a tooth slice. (a) X-ray transmission image. (b) Dark-field images for three different sample orientations $\omega$. (c) Visibility $V(j, k, \omega)$ as a function of $\omega$ for three different sample regions. The dashed black lines are model fits to the measured values. (d) The parameter $\alpha$ describing the proportion of beam that has been scattered. (e) Map of the eccentricity of the fitted local scattering profile. (f) Combined color representation. The eccentricity is mapped onto the image brightness and the angle of orientation of the tubuli in the dentin $\left(\psi_{1}+90^{\circ}\right)$ is mapped onto the image color, using the color wheel displayed in the bottom left corner. Length scale given in (d)-(f). Large version of Fig. 4 shown as Fig. 5.

The results presented in Fig. 4 demonstrate how the parameter $\alpha$ included in the extended model can help us understand the visibility variations in strongly aligned samples. The observed visibility is clearly not sinusoidal and the model presented here is hence necessary to interpret the results. It is noteworthy that this is an extreme case of strongly oriented scattering.

We also see how the directional dark-field measurement provides multiple images containing complementary information. The absorption provides information about the density in the sample, the eccentricity about the degree of ordering, $\psi_{1}$ about the ordering direction and $\alpha$ about the integrated scattering cross section. All of these parameters can be combined to get a better understanding of the investigated samples (Fig. 5).

The orientation of the tubuli in the dentin is important for a number of reasons. The orientation is necessary to understand and model the mechanical properties of teeth. Recently it has also been suggested that future dental filling should mimic the micro and nanostructure of the teeth. ${ }^{23}$ For this to be possible it is necessary to image and understand the nanostructure of the teeth. The use of directional dark-field imaging for this purpose will be particularly interesting when the method is extended into three dimensions. This however still requires the development of novel tensorial $\mathrm{x}$-ray computer tomography reconstruction algorithms, which presently do not exist.

\section{CONCLUSION}

We have developed a simple scattering model to describe the angular variations of the visibility in grating based directional dark-field imaging. The presented model is consistent with previously described methodology while extended to cover also strongly ordered systems which are not well described by the previous model. The novel model was experimentally verified using a fiber mesh sample and a slice of a tooth.

We demonstrated an example of how directional darkfield imaging could be used to determine both the position 

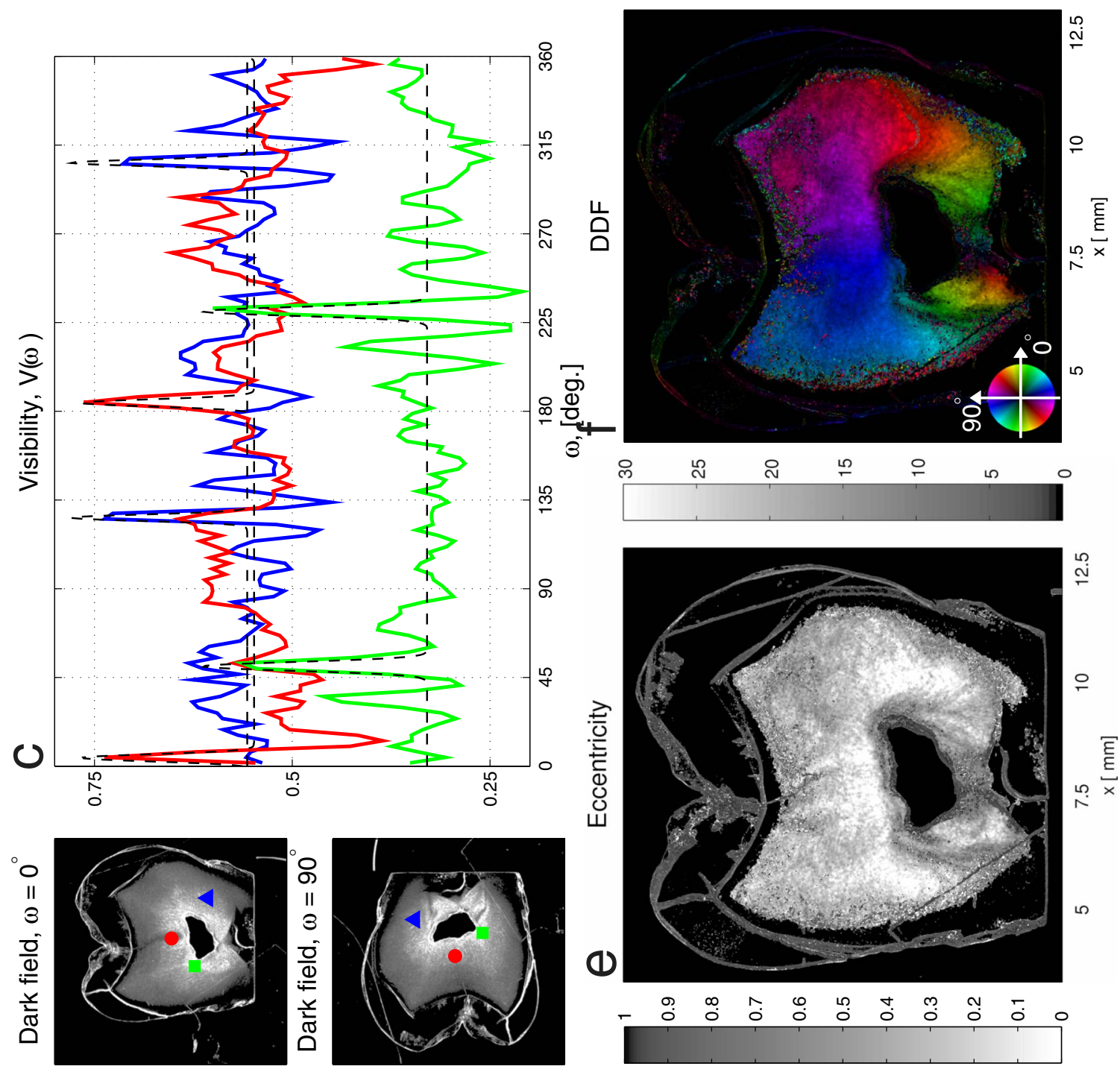

으
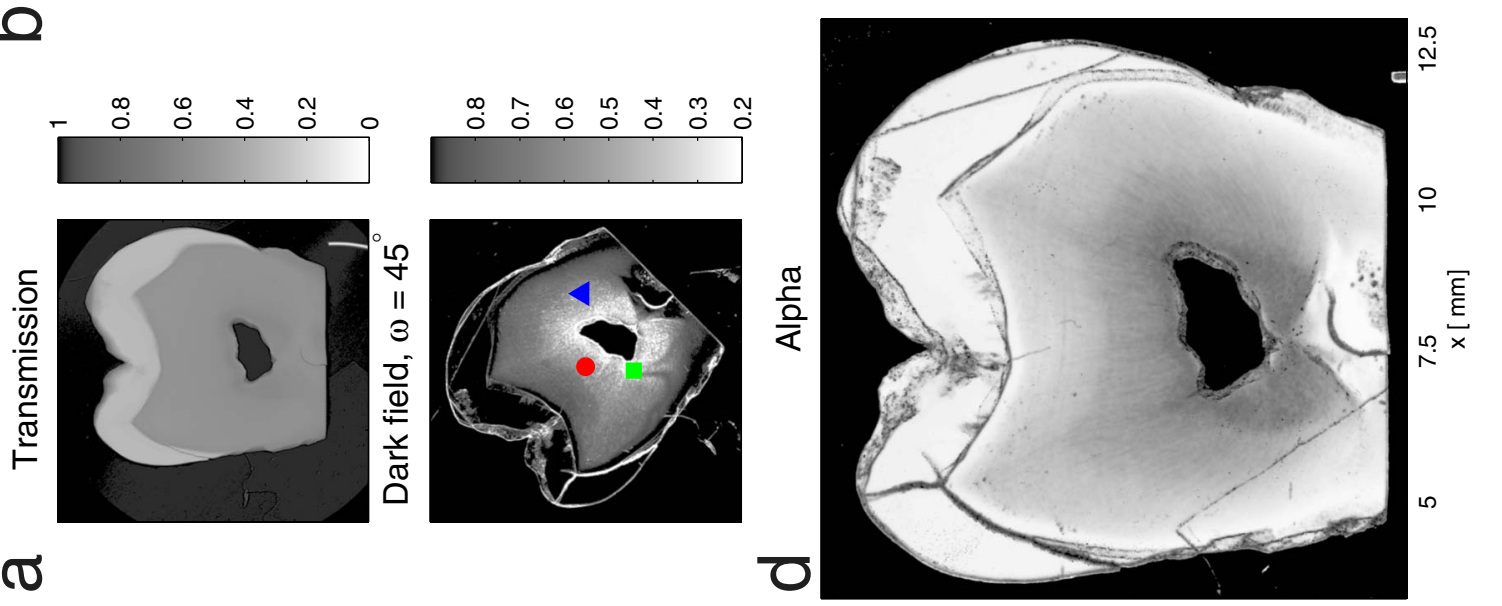

FIG. 5. (Color online) Enlarged version of Fig. 4. Imaging of tooth slice. (a) X-ray transmission image. (b) Dark-field images for three different sample orientations $\omega$. (c) Visibility $V(j, k, \omega)$ as a function of $\omega$ for three different sample regions. (d) The parameter $\alpha$ describing the proportion of beam that has been scattered. (e) Map of the eccentricity of the fitted local scattering profile. (f) Combined color representation. The eccentricity is mapped onto the image brightness and the angle of orientation of the tubuli in the dentin $\left(\psi_{1}+90^{\circ}\right)$ is mapped onto the image color, using the color wheel displayed in the bottom left corner. Length scale given in (d)-(f). 
and orientation of fibers as well as being used to, once calibrated, count the number of overlapping fibers in a given point. The presented model adds to the possible application of directional dark-field imaging for nondestructive testing of for example fiber reinforced materials. It is important to note that the method has previously been demonstrated with a conventional $\mathrm{x}$-ray tube ${ }^{17}$ and one could thus envisage widespread application of the method.

We developed the model further including a term accounting for the not scattered part of the beam. This was used to map the orientation and the eccentricity of the scattering of the tubuli in the dentin. This example demonstrated that directional dark-field imaging can be used for samples exhibiting highly eccentric scattering profiles.

The study of the tubuli in the dentine in teeth will be particularly interesting when the method is extended to three dimensions. As mentioned this however still requires the development of novel tensorial x-ray computer tomography reconstruction algorithms, which presently do not exist.

\section{ACKNOWLEDGMENTS}

We thank Stefan Buser and the clinic for parodontology, endodontology, and cariology of the school for dental medicine (Uni Basel) for sample preparation and Keld Theodor for technical support. F.P. and M.B. acknowledge support through the DFG excellence cluster "Munich-Centre for Advanced Photonics." T.H.J. acknowledges support from the Danish Natural Science Research Council through DANSCATT. This work was supported by the European Synchrotron Radiation Facility (Project No. MI-1001) by allocation of beam time.
${ }^{1}$ C. David, B. Nöhammer, H. H. Solak, and E. Ziegler, Appl. Phys. Lett. 81, 3287 (2002).

${ }^{2}$ A. Momose, Opt. Express 11, 2303 (2003).

${ }^{3}$ T. Weitkamp, A. Diaz, C. David, F. Pfeiffer, M. Stampanoni, P. Cloetens, and E. Ziegler, Opt. Express 13, 6296 (2005).

${ }^{4}$ M. Bech, T. H. Jensen, O. Bunk, T. Donath, C. David, T. Weitkamp, G. Le Duc, A. Bravin, P. Cloetens, and F. Pfeiffer, Z. Med. Phys. 20, 7 (2010).

${ }^{5}$ F. Pfeiffer, T. Weitkamp, O. Bunk, and C. David, Nat. Phys. 2, 258 (2006).

${ }^{6}$ F. Pfeiffer, O. Bunk, C. David, M. Bech, G. Le Duc, A. Bravin, and P. Cloetens, Phys. Med. Biol. 52, 6923 (2007).

${ }^{7}$ M. Bech, O. Bunk, C. David, R. Ruth, J. Rifkin, R. Loewen, R. Feidenhans'l, and F. Pfeiffer, J. Synchrotron Radiat. 16, 43 (2009).

${ }^{8}$ M. Bech, T. H. Jensen, R. Feidenhans'1, O. Bunk, C. David, and F. Pfeiffer, Phys. Med. Biol. 54, 2747 (2009).

${ }^{9}$ F. Pfeiffer, M. Bech, O. Bunk, P. Kraft, E. F. Eikenberry, Ch. Brönnimann, C. Grünzweig, and C. David, Nature Mater. 7, 134 (2008).

${ }^{10}$ F. Pfeiffer, M. Bech, O. Bunk, T. Donath, B. Henrich, P. Kraft, and C. David, J. Appl. Phys. 105, 102006 (2009).

${ }^{11}$ B. Schwarzschild, Phys. Today 61(3), 12 (2008).

${ }^{12}$ M. Bech, Ph.D. thesis, University of Copenhagen, 2009.

${ }^{13}$ Z.-T. Wang, K.-J. Kang, Z.-F. Huang, and Z.-Q. Chen, Appl. Phys. Lett. 95, 094105 (2009).

${ }^{14}$ G.-H. Chen, N. Bevins, J. Zambelli, and Z. Qi, Opt. Express 18,
12960 (2010).

${ }^{15}$ M. Bech, O. Bunk, T. Donath, R. Feidenhans'1, C. David, and F. Pfeiffer, Phys. Med. Biol. 55, 5529 (2010).

${ }^{16}$ W. Yashiro, Y. Terui, K. Kawabata, and A. Momose, Opt. Express 18, 16890 (2010)

${ }^{17}$ T. H. Jensen, M. Bech, O. Bunk, T. Donath, C. David, R. Feidenhans'1, and F. Pfeiffer, Phys. Med. Biol. 55, 3317 (2010).

${ }^{18}$ T. Weitkamp, I. Zanette, C. David, J. Baruchel, M. Bech, P. Bernard, H. Deyhle, T. Donath, J. Kenntner, S. Lang, J. Mohr, B. Müller, F. Pfeiffer, E. Reznikova, S. Rutishauser, G. Schulz, A. Tapfer, and J.-P. Valade, Proc. SPIE 7804, 780406 (2010).

${ }^{19}$ C. David, J. Bruder, T. Rohbeck, C. Grünzweig, C. Kottler, A. Diaz, O. Bunk, and F. Pfeiffer, Microelectron. Eng. 84, 1172 (2007).

${ }^{20}$ E. Reznikova, J. Mohr, M. Boerner, V. Nazmov, and P. Jakobs, Microsyst. Technol. 14, 1683 (2008).

${ }^{21}$ Due to noise, a minimal visibility of 0.06 has been used in the fits. We would expect a noise level of this magnitude and thus believe this is not an effect of the not scattered part of the beam.

${ }^{22}$ The human tooth was an extracted nonrestored molar tooth of the fourth quadrant. A saw (Exact Apparatebau GmbH, Norderstedt, Germany) equipped with a $0.2 \mathrm{~mm}$ cutting diamond band was used to make a 500- $\mu$ m-thick slice parallel to the tooth axis.

${ }^{23}$ H. Deyhle, O. Bunk, S. Buser, G. Krastl, N. U. Zitzmann, B. Ilgenstein, F. Beckmann, F. Pfeiffer, R. Weiger, and B. Müller, Proc. SPIE 7401, 74010E (2009). 